\title{
Practical, Appropriate, Empirically-Validated Guidelines for Designing Educational Games
}

\author{
Conor Linehan, Ben Kirman, Shaun Lawson \\ Lincoln Social Computing Research Centre \\ University of Lincoln, LN6 7TS, UK \\ \{clinehan, bkirman, slawson\}@1incoln.ac.uk \\ $++441522837084$
}

\author{
Gail G Chan \\ Oxford Brookes University \\ Jack Straws Lane, Oxford, OX3 OFL, UK \\ gchan@,brookes.ac.uk \\ $+447576347701$
}

\begin{abstract}
There has recently been a great deal of interest in the potential of computer games to function as innovative educational tools. However, there is very little evidence of games fulfilling that potential. Indeed, the process of merging the disparate goals of education and games design appears problematic, and there are currently no practical guidelines for how to do so in a coherent manner. In this paper, we describe the successful, empirically validated teaching methods developed by behavioural psychologists and point out how they are uniquely suited to take advantage of the benefits that games offer to education. We conclude by proposing some practical steps for designing educational games, based on the techniques of Applied Behaviour Analysis. It is intended that this paper can both focus educational games designers on the features of games that are genuinely useful for education, and also introduce a successful form of teaching that this audience may not yet be familiar with.
\end{abstract}

\section{Author Keywords}

Education, Educational Games, Serious Games, Games Design, Applied Behaviour Analysis, Psychology, Behavioural Psychology.
Note: This is the author's version of the work. It is posted here by permission of ACM for your personal use. Not for redistribution. The definitive version was published in Proceedings of the 29th of the international conference extended abstracts on Human factors in computing systems.

\section{INTRODUCTION}

There has recently been a great deal of interest in harnessing the motivational qualities of computer games in order to create powerful, engaging educational tools $[22,49,48,46]$. However, little is known about how, exactly, games achieve these motivational qualities. Furthermore, there are very few practical recommendations made on how to recreate the motivation seen in entertainment games when designing educational games. Most literature that has been published on the design of games for education is purely descriptive and discusses why games should be good learning tools, rather than what can be done to make sure that they are. Perhaps as a consequence, the majority of educational games can be described as chocolate-covered broccoli [9] neither fun, nor of educational benefit.

The intention of this paper is to provide a clear, practical framework for designing engaging game mechanics in educational games, based on the successful, empirically validated teaching methods developed by behavioural psychologists. We are motivated by the findings that whilst there is very little evidence of computer games outperforming traditional teaching methods in terms of contributing towards students successfully achieving education outcomes, there is a great deal of evidence of behavioural teaching programmes doing so. Crucially, the methods that computer games designers and behavioural psychologists have developed for teaching appear very similar to each other in many ways. Both computer games and behavioural teaching programmes are approaches to education that reject the traditional lecture format and use novel, creative methods of structuring the education process, centred on personalised instruction.

In this paper we will first introduce the argument for using games as educational tools and the problems that are inherent in doing so. We will then discuss the processes involved in designing engaging computer games, before addressing the problem of merging the disparate goals of education and game design. We will then suggest that Applied Behaviour Analysis (ABA), the method of teaching developed by behavioural psychologists, can provide a foundation for the design of educational games, while maintaining those aspects of entertainment games that are 
crucial to player motivation. We will conclude by providing a step-by-step guide in designing an educational game on the basis of ABA.

\section{GAMES AS TOOLS FOR EDUCATION}

Student motivation is a key predictor of successful educational outcomes $[38,39]$. Indeed, one of the most basic and important predictors of student achievement is the amount of time a student spends engaged in learning (or time-on-task; $[1,27,20,57])$. Traditional mainstream education has been criticised by many as being poor at motivating all but a small minority of learners [55]. Much criticism has been directed towards the lecture-based structure that is still ubiquitous in educational institutions today [45,48]. Critics have typically asserted that the primary advantage of the lecture format is economical rather than pedagogical [41]. What appears to be needed is a medium that provides similar cost-effectiveness as the lecture format, but with the ability to retain rather than alienate learners [41].

Video games have emerged as a technology that specialises in, and is hugely successful at, engaging and motivating users to spend time-on-task $[15,22,41]$. It is clear that people are highly motivated to engage in these games [48]. In addition to the apparent motivational qualities of computer games, many have commented on how learning is an essential part of successfully playing a game $[22,23,30,48]$. Indeed, it could be argued that all commercial games are educational, as they train players to learn the skills needed for gaining success within that game. Essentially, it seems that games designers have hit on profoundly successful methods of getting people to learn and to enjoy learning $[22,30]$.

Given that games specialise in engendering motivation, are hugely popular and already require players to learn, it should be no surprise that the creation of games that provide some form of learning experience for the player has been proposed. Educational games appear to offer the potential to improve learner motivation, time-on-task and, consequently, learning outcomes.

\section{Existing games are not particularly successful}

While it seems apparent that games have the potential to function as valuable teaching tools, there is very little evidence that they 1) produce reliable, valid and longlasting educational outcomes, or 2) do so better than traditional education structures. There is very little empirical work published that investigates the educational potential of games in any rigorous manner, and less still that measures the learning outcomes of games compared to other teaching methods [41]. Indeed, review articles $[15,44]$ tend to focus on pointing out reasons why games have the potential to constitute valuable learning tools, rather than reviewing any empirical evidence.

O'Neill et al. [46] conducted a thorough review of articles published on educational computer games in the previous
15 years. They found that, despite the thousands of articles available, only 19 presented a rigorous quantitative or qualitative analysis of educational outcomes from computer games. Of those 19 articles, findings regarding the educational benefits of games were mixed, which echoes the findings of other commentators $[14,24,49]$. Furthermore, in cases where positive educational outcomes have been found, it is possible that these can be attributed to the instructional design of the educational programme and not to games as a medium (see [12] for a discussion on this point). O'Neill et al., conclude that, "the evidence of potential is striking, but the empirical evidence for effectiveness of games as learning environments is scant" (p. 468).

It is important to note that although reviews have pointed to a lack of convincing evidence for games as educational tools, this does not mean that there haven't been any games that have produced positive educational outcomes. For example, Lee et al. [31] found that students completed 3 times as many maths problems over 19 days using a computer game than they normally did using just worksheets. McLean et al. [43] found favourable results for games in comparison to other teaching methods, across a range of games that taught subjects as diverse as geology and biology. A randomised control trial of a diabetesmanagement game was found to reduce hospital visits for children aged 8-16 who played it [32]. Indeed, every month there are growing numbers of studies that demonstrate how games can facilitate learning.

However, despite this growing evidence base, it is questionable whether any greater level of understanding on how to best produce learning outcomes through game-based teaching programmes is being generated. Without a solid theoretical framework through which to design studies and interpret results, it is very difficult to differentiate which elements of a successful game were essential in producing those successful outcomes and which were not necessary.

Factors that predict effectiveness of educational games Given the apparent consensus that games should be effective tools for education, and, further, that some studies have found positive effects for games on educational outcomes, it is surprising to discover that there have been very few concrete, empirically validated suggestions on how to design successful educational games $[15,21,24,41,44,46]$. In fact, a large number of factors have been suggested as potentially important, but few have been demonstrated conclusively. For example, commentators have suggested that factors such as fun [2], flow, engagement [30], feedback [40, 41], goals $[15,56]$, problem solving [23], game balance and pacing [23], interesting choices [51] and fantasy narrative [38,39], among many others are essential to the success of a game. Indeed, perhaps the most influential book in the field of educational gaming [22] mentions 36 possible ways that people can learn from games. What is missing is the 
evidence of components that $d o$ reliably predict successful outcomes.

One exception to this analysis appears to lie in the concept of Intrinsic Learning ([25]; also referred to as procedural rhetoric [8]). Intrinsic learning requires the embedding of learning outcomes of a teaching programme within the mechanics of a game. It appears crucial that the task learned in the game maps directly on to the challenge faced in the real world. Habgood [25] investigated experimentally the importance of integrating learning content with the mechanics of a game. Specifically, in two studies, he found that a game in which learning was intrinsic to game play was motivationally and educationally more effective than an almost identical game in which learning was not intrinsic to game play. Thus it appears that we have at least one coherent, experimentally verified rule for designing educational games; a successful educational game must integrate the learning with the game play mechanics, rather than as an addition to the game play mechanics.

In summary, while it may prove useful for games designers to give suggestions on game features that enhance engagement, if they are not specific enough to test empirically they are of very little use to other designers. What is necessary in order to advance the understanding of how to build successful educational games is a rigorous framework for defining parameters that can be experimentally validated. We propose that applied behaviour analysis (ABA; [13]) may form an ideal framework for both guiding the design of educational games and conducting the basic research on how to teach successfully via games.

\section{DESIGNING COMPUTER GAMES}

One of the key mistakes made by educational games designers lies in focusing too heavily on educational content to the detriment of the gameplay [25]. The reason why games have been proposed as good educational tools is that they are seen as intrinsically motivating. However, that does not mean that everything which is called a game, or looks like a game, will have those motivating qualities. Simply translating a standard textbook-style educational programme into a point-and-click computer programme, does not constitute game design. Rather, a similar approach must be taken to designing an educational game as that is taken to entertainment games.

In order to build a successful educational game, it is crucial to understand what is involved in designing a traditional computer game. From a review of the literature on game design [7,30,36,51] the following features appear important to most games:

Games typically present the player with a series of short, medium and long-term goals. Indeed, Swartout and van Lent [56] found that goals of different levels help motivate learners to continue playing.
Games typically require the player to take some actions or decisions in order to reach those goals [51].

Games typically excel at providing immediate, appropriate and specific feedback to players. This feature is at the heart of the motivation, sustained attention, learning and fun experienced by game players $[36,40,41]$.

Games often have a complex system for presenting players with rewards for achievement.

Games methodically teach players the skills needed to meet complex challenges. Long, complex tasks are broken down into short, simple components. These components are trained individually before being chained together $[22,23,30]$.

Generally, players are expected to demonstrate excellent performance of a skill before they can advance to using that skill in a more challenging environment. Complex tasks, then, simply require the chaining together of these previously learned simple skills.

Where games present the player with options for taking action, no one action should be obviously correct, while others are obviously incorrect $[22,30,48,51]$.

Table 1: List of features seen in most entertainment games.

Of course, this list is in no way exhaustive, and including these seven features will not guarantee a successful game. However, these features are observed in a lot of successful entertainment games, thus implying that they are useful in the engendering and maintaining of player motivation. So, when designing educational games, it appears important to take a pedagogical approach that allows for the inclusion of those features.

\section{INTEGRATING THE GOALS OF EDUCATION AND GAME DESIGN}

In order to design successful educational games we need to adopt an educational framework that is both successful and congruent with the goals of game design. We also need a method of instruction that takes greatest advantage of the benefits that medium of computer games offer; their ability to teach in a one-to-one manner, to adapt to the performance of each individual player, to deliver timely and specific feedback to players in a controlled manner, and to motivate players of a wide range of knowledge or skill levels. We propose that Applied Behaviour Analysis (ABA), as a framework, fulfills all of these requirements.

ABA programmes, such as Precision Teaching [33,34,35], Direct Instruction [16], and the Personalized System of Instruction [28] are methods for teaching based on the findings of behavioural science. They are typically delivered on a one-to-one basis and reject the lecture format, relying on the teacher more as a coach. They are 
designed on the assumption that learning is maximised when high performance targets are set and teaching is focused on the individual. Indeed, unlike in traditional education, the passing criterion in behavioural education is not $40 \%$, but typically somewhere around $90 \%$. If the learner does not reach this stringent passing criterion, they are required to repeat the programme until they do reach it.

\section{ABA programmes are highly successful}

There has been a great deal of empirical support for the effectiveness of behavioural teaching programmes. Indeed, they have been extremely successful wherever implemented, from university modules [52] to secondary school, [45] primary school [33,34], driver education programmes [4] and challenging populations [11]. Behavioural teaching methodologies have been particularly successful as early interventions for children diagnosed with autistic spectrum disorders [37].

Despite the empirical evidence for the effectiveness of behavioural teaching methods, they have not yet been widely adopted within mainstream education in any country to date. A number of suggestions (i.e., $[4,35,45,52]$ ) have been put forward to explain this situation. For example, educators may be hesitant to adopt instructional methods that are contrary to traditional pedagogical systems or are difficult to fit into the traditional academic calendar [52]. Behavioural strategies require substantial planning, preparation of resources and training, which many educators have been unwilling to undertake [4].

One of the core barriers to acceptance, according to Lindsley [35], is the focus on repetition and discipline within behaviour methodologies and how these don't fit well with current views commonly held by educators that learning should be fun. He draws a comparison between the attitudes of educators and those who coach in athletics and the performing arts. "It is amazing that educators and the public accept the need for disciplined regular daily practice in the performing arts and in athletics, yet reject it in academics."

Crucially, computer games appear ideally suited to circumvent a large number of the factors that are holding back the uptake of these highly effective teaching programmes in mainstream education. The use of computer games require little extra training for the teacher, since a well designed game itself teaches the user how to play it. Games can be fit within the traditional academic timetable, they require no resources other than the game itself, and they motivate players to engage in exactly the type of disciplined, repetitive rehearsal that Lindsley [35] suggested as crucial to success in education.

\section{ABA is similar in structure to games}

ABA programmes, have structures that resemble computer games in important ways. For example, in a Precision Teaching programme [33,35], the teacher defines each individual target behaviour that the student needs to improve on. They also define a target frequency of that behaviour, and clearly state the rewards for reaching that target. For example, the goal may be to answer forty 12times-tables problems in a 30 second period. The student practices the behaviour until the specified target is met. Once performance targets have been met, the student obtains the pre-defined reward and the teacher sets a new task. Students record their own performance under supervision of teachers on specially designed charts. From viewing these charts, students have constant access to feedback on their performance relative to previous sessions.

Interestingly, highly engaging games typically have clearly specified and measurable goals (such as to level-up), require a great deal of repetition of skills in order to reach that goal (fighting numerous similar enemies), are often conducted under time constraints, have clearly specified rewards for reaching the specified goal (stronger player/more weapons/access to new levels) and provide constant feedback from the game state on how successfully the player is performing. The similarities to behavioural teaching programmes are striking. In addition, successful games pay a great deal of attention to the rate in which complexity is increased over the course of game levels and to the balance and pacing of player advancement through these levels. These issues of rates, balance and pacing appear to precisely parallel the process that the behaviour analyst undertakes in defining larger programmes for students.

\section{Has this approach to educational games been undertaken before?}

Some researchers have already adapted and used games within behavioural education programmes. For example, Foxx et al. [19] adapted a commercially available board game to train social and vocational skills to developmentally delayed adults. The game contingencies increased social/vocational skills in all targeted areas. Medland and Stachnik [42] successfully used a game to improve the classroom behaviour of fifth grade students. The game reduced the dependent measures from their baseline rate by almost $99 \%$ for one group and $97 \%$ for the other. Bay-Hinitz, Peterson and Quiltich [3] used cooperative games to modify the aggressive and co-operative behaviours of young children. Cooperative behaviour increased and aggression decreased during cooperative games and these behaviours generalised to subsequent freeplay sessions.

It appears that behaviour modification and teaching programmes can, and have, been delivered effectively via games. Indeed, Bay-Hinitz [3] point out that there is a practical advantage to delivering behavioural interventions via games; "instructing children in the use of particular games is a simple strategy, particularly when compared to training a teacher to carry out a specific treatment plan using contingent reinforcement for certain responses" ( $p$. 445). However, these findings have made little impact on the field of educational games in general. 
In summary, it appears that $\mathrm{ABA}$ programmes are both highly successful methods for teaching, and also resemble games in a number of important ways. In fact $A B A$ programmes appear ideally suited to taking advantage of the benefits that the medium of computer games offer; they excel in teaching in a one-to-one manner, they are adaptive to the performance of each individual learner, they are based on delivering timely and specific feedback to learners in a controlled manner, and they have been used to motivate players of a wide range of knowledge or skill levels. As games and ABA programmes appear to share all of these important features, it should be possible to build games based on ABA principles without losing the motivation typically elicited by entertainment games.

\section{ABA AND THE DESIGN OF EDUCATIONAL GAMES}

In this section will attempt to explain some basic steps taken by behaviour analysts in designing educational programmes, and point out how they can be implemented in the design of educational computer games. The steps presented below should, on their own, be enough to improve the chances of games genuinely helping learners to achieve their learning outcomes. These steps can be broken into three categories; a) defining and measuring behaviour, b) recording and analysing behaviour change, c) presenting corrective feedback, and d) dynamically adapting to student performance.

\section{Defining and measuring behaviour}

\section{Selecting and defining target behaviours}

The most basic and important step in the design of any educational programme is to clearly define the intended learning outcomes of that programme. These outcomes must be defined as clearly, objectively observable behaviours, and it must be possible to provide a specific definition of when a learner has and has not reached that learning outcome. For example, rather than an outcome of 'knowledge of 12-times tables' it should be defined as, the ability to solve any twenty randomly presented 12-times tables problems in a 30 second window. This definition is testable and is possible for a computer to evaluate as correct or not.
Similarly to the concept of intrinsic learning suggested by games designers [8,25], behaviour analysts insist that the "behaviour chosen .... must be the behaviour in need of improvement, not a similar behaviour that serves as a proxy for the behaviour of interest, or the subjects verbal description of the behaviour" ([13], p.16). So, whether or not participants have reached a learning outcome should not be judged by their answers to a questionnaire (unless the learning outcome is to improve the learners ability to answer questionnaires). Rather, whether they have reached that learning outcome or not should be observable from the action of the player as they are playing.

Essentially, instruction should not be designed as blocks of play and tests. The play, itself, should be the behaviour that is examined. This is especially important in a computer game, where a teacher is not present to interpret behaviour. So, if the game has been designed to teach industrial chemistry, and a learning outcome is an understanding of the chemical reactions necessary for the extraction of iron from iron ore, the game should take place at the molecular level. It is not appropriate to create a game where people can throw random chemicals into a furnace, then observe the outcomes and answer a multiple choice questionnaire.

$\mathrm{ABA}$ programmes, like games, break complex tasks into simpler component tasks and ensure that learners can perform those simpler tasks before requiring performance of the complex skill. So, the designer must clearly define not only the ultimate learning outcome of the programme, but also the series of steps that learners must reach on their way to that goal. In this way, a hierarchy of learning outcomes is created, where knowledge of the simplest concepts and processes are taught first, and knowledge and performance are built methodically. Once the player has passed a challenge and demonstrated a knowledge of a particular concept, they are immediately presented with a more complex challenge that builds upon that newly acquired information.

\section{Measuring Behaviour}

Measurement refers to the process of assigning numerical values to observed behaviour. Above, we mentioned the

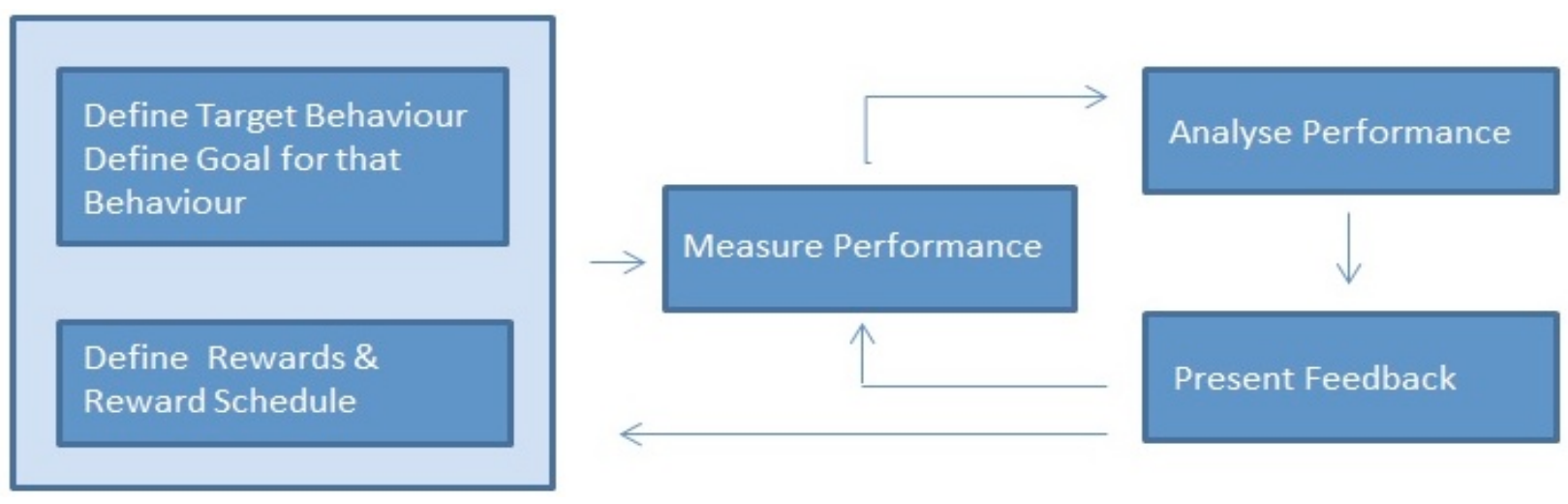

Figure 1. Diagram of the processes involved in an ongoing ABA programme. 
importance of ensuring that the skill that is the focus of the programme is exactly the same as the skill being measured. This is particularly important when designing educational computer games, as it will allow us to automate the process of evaluation and feedback.

We must also consider the units we are measuring. In traditional educational settings it is normal to report the number of tasks that have been performed correctly and the number that have been performed incorrectly. ABA programmes focus heavily not only on accuracy, but on temporal aspects of performance. So, tasks are typically carried out within time constraints. Behaviour analysts have found that measures which include temporal components are a more accurate method for judging how comfortable, or fluent the learner is with the material than simple measures of accuracy [13]. So, requiring accurate performance within strict time constraints is a proven method for ensuring that learners are familiar with the material, and also reflects a commonly used game mechanic.

\section{RECORDING AND ANALYSING BEHAVIOUR CHANGE}

\section{Recording data}

Closely related to the process of measuring behaviour, is that of recording those measurements in a way that is easy for both the learner and instructor to understand. Behaviour analysts record every single instance of every target behaviour. For example, in a spelling exercise, the position of each letter in a word is checked and marked whether it is correct or not. These raw data points are then entered on line graphs, called celeration charts, which allow both the teacher and learner to view easily any changes in the learners' performance of that behaviour. In a computer game, these data points must be recorded in a way that is easy for the game application to read and analyse.

Interestingly, the delivery of ABA programmes via computer games allows for a great deal of innovation in terms of recording and presenting data in ways that are useful for both educators and learners to understand. Celeration charts have been developed for their ease of use for the instructor, but computers have a far greater capacity for manipulating data. While it will still be essential to record every data point and present them in a meaningful way, just how that data is presented is open to the creativity of the game designer. For example, games typically track performance through a representation of health, lives, level, and other such metrics. This ability to present data in an engaging manner may be one of the key ways that computer games can actually improve upon existing ABA programmes.

\section{Analysing behaviour change}

The key metric used by behaviour analysts in monitoring the success of learners is the change in their behaviour over time. Essentially, once a learning outcome has been defined, the behaviour analyst continually measures the learner performing that behaviour and examines whether or not the learner is approaching that outcome.

Consider the example of a coach who is teaching a player to improve their ability to score 3-point shots in basketball. The coach will allow the player to shoot freely, marking down the number of baskets the player makes out of ten shots. The coach then instructs the player in how to improve their technique before watching them practice. Again, the coach observes and marks down on a chart how many are scored. This continues, until the player reaches their target, with the coach intervening only occasionally.

Automating the process of analysing behaviour change is simple, once the preceding steps of defining, measuring and recording behaviour have been carried out in a methodical fashion. In computer games, it may not be always possible to take a true baseline - if the taking of baseline data does not seem congruent to the game design, then performances on early attempts can be compared with performance on later attempts in order to evaluate whether change in behaviour is happening. Computer games can do exactly this type of analysis, without the need for complex machine learning algorithms.

\section{Providing corrective feedback}

\section{Presenting feedback}

Feedback is presented to learners in most educational programmes in order to give them information regarding how closely their current level of performance matches their goals. The intention is to guide the learner towards excellent performance.

The process whereby performance-related feedback alters the behaviour of learners has been referred to, by behavioural psychologists, as operant conditioning [53,54]. Through investigations of operant conditioning, behavioural psychologists have demonstrated how feedback should be presented in order to maximize the attention and motivation of learners [10]. These findings are directly relevant to the design of educational games. A brief explanation of how these concepts are currently used in games is presented below.

Positive Reinforcement describes a situation where the presentation of a stimulus as a consequence of an instance of behaviour makes that behaviour more likely to occur in that context in future. For example, gaining experience points and gold pieces for killing a goblin may make goblin-slaughter the more likely response to future goblin encounters.

Negative Reinforcement describes a situation where the removal or termination of an existing stimulus (or existing aversive condition) as a consequence of an instance of behaviour makes that behaviour more likely to occur in that context in future. Negative reinforcement is manifest in games where players are forced to re-start from the 
beginning of a level or stage when they die - not wanting to waste time replaying the easy parts of the same level overand-over is a powerful motivator.

Positive Punishment describes a situation where the presentation or addition of a stimulus as a consequence of an instance of behaviour makes that behaviour less likely to occur in that context in future. For example, in modern First-person Shooter Games, running aggressively into battle with machine guns firing and little regard to tactics is typically punished by the player's' rapid death.

Negative Punishment describes a situation where the removal or termination of a stimulus as a consequence of an instance of behaviour makes that behaviour less likely to occur. For example, in role-playing games, upon the player's death they are deducted an amount of previously collected experience points.

Table 2. Explanation of how operant conditioning processes work in computer games.

Both engaging games and successful ABA programmes use these basic processes in combination in order to ensure that the game is able to provide consistent, appropriate and specific feedback to the player, and to guide them towards performing at a high skill level. When you wish a player to learn a particular skill or strategy while playing your game, it appears that adopting the following approach is useful; a) offering a variety of rewards for correct performance, b) offering persistent negative consequences for poor performance, which the player will work to avoid, and c) directly presenting negative consequences when the player does something that you do not want them to do.

\section{Evaluating the effectiveness of feedback}

In order to apply these techniques successfully, it is crucial to understand the difference between a 'reward' and a reinforcer. A reward is any stimulus given to a player on the assumption that it will increase the likelihood of the rewarded behaviour being repeated in future. However, simply providing game players with extra points, new items, or access to new levels is of little value unless the game actually checks to see whether the player has learned from the reward.

Behavioural psychologists continually test whether the rewards they offer produce changes in the target behaviour (i.e. learning). If the targeted behaviour does increase as a consequence of the delivery of a reward stimulus, then the game can classify that stimulus as a reinforcer, and use it later when necessary [53]. So, a reward simply refers to anything presented as a consequence of desired performance; a reinforcer is an example of one of those events that has been demonstrated to work. The same principle applies to punishers.

As there are very few (if any) stimuli that function as a reinforcer or punisher for all people at all times, it is necessary that the game should be able to evaluate the impact of the different rewards that it offers in order to evaluate whether the rewards provided by the game are ones that the player, as an individual, is personally motivated to obtain. Indeed, there should be different types of rewards that players can work for at any time and the game should include some simple way of evaluating which rewards are most reinforcing for each player.

Fortunately, behavioural psychologists have developed useful techniques for evaluating the relative reinforcing strength of different rewards, and it appears that these techniques may be ideal for use in computer games. Herrnstein [26] found that when two or more behaviour options are available, behaviour can be explained quite well via a mathematical formula that he called the 'matching law.' Understanding the matching law can help games designers create uniquely adaptive and engaging games. Herrnsteins algorithm allows us to monitor the relative attractiveness of each of the various reinforcers on offer with a given game.

Through continuously monitoring a players behaviour, a game (educational or otherwise) can automatically calculate which rewards are eliciting the most work from that player (i.e., which are most reinforcing). In an entertainment game such as Fallout 3 [6], for example, through monitoring player activity, the game could easily identify that a given player has spent twice as much time or effort doing missions for which they will receive rare items, as those for which they receive 'karma' points. Using the Matching Law to interpret this data, the game could deduce that rare items are twice as reinforcing for that player as karma points. Thus, using this simple strategy, it is possible for games to dynamically evaluate the reinforcing strength of each of the available game rewards for each individual player player, and make adjustments to its own reward system in order to exploit this data. A similar process would be very useful for maintaining the engagement of educational games players.

\section{Scheduling rewards}

Of huge importance to educational game design is the question of how best to maintain the attention and motivation of the learner. Behaviour analysts have found that in order to maintain behaviour, we must carefully consider not only what to use as a reward, but also how and when learners should gain access to those rewards. It is not sufficient to offer a reward after every action that a user takes. Rather, in order to sustain attention over a period of time, it is necessary to manipulate either the number of responses required, or the time elapsed before reinforcement is delivered for a particular behaviour [18]. Different schedules are appropriate in different contexts, depending on the type of behaviour you wish to engender in the user. This process has been examined extensively in the behaviour analysis literature under the term 'schedules of reinforcement' $[10,18]$. Below are four schedules 
commonly used in both education settings and computer games.

Fixed Ratio schedules deliver reinforcement after every nth response. For example, FR5 schedules provide reinforcement consistently after every fifth response. This schedule produces a high, steady rate of responding with a brief pause after the delivery of the reinforcer.

Variable Ratio schedules are similar to FR schedules, with the exception that, rather than being predictable, the number of responses required for reinforcement oscillates around a mean. This type of schedule creates a high and steady rate of responding and is typically the most economical; a lot of work can be generated by few instances of reinforcement.

$A$ Fixed interval is a schedule where only the first response after a specified amount of time has elapsed is rewarded, while premature responses are not reinforced.

Variable Interval schedules are similar to FI schedules, with the exception that the time for which reinforcement is unavailable oscillates around a mean, rather than being predictable.

Table 3. Explanation of four different schedules of reinforcement.

Behaviour analysts use combinations of these schedules in order to motivate learners. Indeed, the literature [10,13,18] suggests that if people have a consistent history of being reinforced for their efforts, the workload required to reach those same rewards can be increased gradually over time without losing the motivational effects of those rewards. It is not necessary, or even good practice, to keep rewarding every action. Behavioural Psychologists refer to this technique as 'schedule leaning.' Indeed, the technique is also observed in computer games, where the first few tasks that a player completes are reinforced through new items, new skills and leveling-up. However, as the player progresses and spends more time playing the game, the number of actions needed to produce a reinforcer are increased.

\section{Adapting to student performance}

Both games and ABA programmes are built on the assumption that the challenges presented should be appropriate to the users' skill level. There should always be the opportunity for reinforcement, regardless of the level of ability that a learner initially demonstrates. In order to ensure that this is the case within an ABA-based educational game, that game must be able to analyse the players performance and adapt in order to consistently present appropriate challenges. This appears to be quite a challenge, but can be done quite effectively if the previous steps have been taken carefully.
Firstly, clearly defined goals, each representing components of the ultimate target behaviours, mean that present behaviour can always be compared to a relevant goal. If goals are met quickly, the player should advance at a fast pace. If goals are not met after repeated attempts, the player should be required to repeat the simpler component tasks. In this way, the player will always be striving towards achievable goals and the game will not present any challenge without providing the player with the skills required to pass it.

Secondly, as the player advances through the game at a quick pace, the game can recognize this and begin presenting rewards on a leaner schedule. The increased scarcity of rewards as the game progresses functions to sustain behaviour.

Thirdly, using the matching law, the game can evaluate the relative reinforcing strength of the various rewards on offer in the game. This information can be used in a variety of different ways, depending on what effect on player behaviour is necessary. For example, they can offer proportionally more of the more attractive rewards (adapting to individual differences), reduce the frequency of those rewards over time (leaning the schedule), or adjust the amount of work required to achieve each of the available awards, in order to ensure that players are motivated to experience each element of the game.

\section{CONCLUSION}

In this paper we have identified that while computer games appear to represent an exciting new paradigm in education, there is a lack of empirical evidence for their effectiveness. We suggest that the current lack of success of these tools is due to a lack of theoretical and methodological rigor in the design of educational games. There is a misunderstanding of the game features that must be included in educational games and the teaching methodologies that are compatible with gameplay.

We propose that Applied Behaviour Analysis (ABA), a personalised method of teaching that has been demonstrated as highly successful wherever implemented, appears uniquely positioned to take advantage of the benefits that the medium of computer games offer; their ability to teach in a one-to-one manner, to adapt to the performance of each individual player, to deliver timely and specific feedback to players in a controlled manner, and to motivate players of a wide range of knowledge or skill levels.

Finally, we have laid out some steps that are central to the design of any ABA programme. Including these steps in the design of any educational game will ensure that the game teaches in a way that has both been demonstrated as effective and is also appropriate to the medium of computer games. 


\section{ACKNOWLEDGEMENTS}

This work was carried out as part of the "Leonardo" project "Learn to Lead" funded by the EU Lifelong Learning Program (http://www.learn2lead.unina.it).

The authors also wish to acknowledge informal discussions held with Bryan Roche at the National University of Ireland, Maynooth, and with Denis O'Hora and Ian Stewart at the National University of Ireland, Galway, which have been useful in formulating the ideas presented in this paper.

\section{REFERENCES}

1. Admiraal, W., Wubbels, T., and Pilot, A. College teaching in legal education: Teaching method, students' time-on-task, and achievement. Research in Higher Education, 40 (1999), 687-704.

2. Amory, A., Naicker, K., Vincent, J., and Adams, C. The use of computer games as an educational tool: identification of appropriate game types and game elements. British Journal of Educational Technology, 30 (1999), 311-321.

3. Bay-Hinitz, A.K., Peterson, R.F., and Quilittch, H.R. Cooperative games: a way to modify aggressive and cooperative behaviors in young children. Journal of Applied Behavior Analysis, 27 (1994), 435-446.

4. Bell, K. E., Young, K. R., Salzberg, C. L., and West, R. $\mathrm{P}$. High school driver education using peer tutors, direct instruction, and precision teaching. Journal of Applied Behavior Analysis, 24 (1991), 45-51.

5. Bellotti, F., Berta, R., De Gloria, A., and Primavera,1. Enhancing the educational value of video games. $A C M$ Computers in Entertainment, 7, 2 (2009), Article 23.

6. Bethesda Game Studios (2008). Fallout 3. [PC], USA: Bethesda Softworks.

7. Björk, S., \& Holopainen, J. Patterns in game design. Hingham, MA: Charles River Media, 2004.

8. Bogost, I. Persuasive Games. Cambridge, MA: MIT Press, 2007.

9. Bruckman, A. Can Educational Be Fun? Game Developer's Conference, (1999).

10. Catania, C. A. Learning, (4 ed). Cornwall-on-Hudson, NY: Sloan Publishing, 1998.

11. Christophersen, E. R., \& Mortweet, S. L. Treatments that work: Empirically supported strategies for managing child behavior problems. Washington, DC: American Psychological Association, 2001.

12. Clark, R.E. Reconsidering research on learning from media. Review of Educational Research, 53, 4 (1983), 445-459.

13. Cooper, J. O., Heron, T. E., and Heward, W. L. Applied Behavior Analysis, (2 ed.). NJ: Prentice Hall, 2006.

14. De Aguilera, M. and Méndiz, A. Video Games and Education (Education in the Face of a "Parallel
School"). ACM Computers in Entertainment, 1, (2003), Article 01.

15. Dondlinger, M.J. Educational Video Game Design: A Review of the Literature. Journal of Applied Educational Technology, 4 (2007), 21-31.

16. Engelmann, S., Becker, W. C., Carnine, D., and Gersten, R. (1988). The direct instruction follow through model: Design and outcomes. Education and Treatment of Children, 11 (1988), 303-317.

17. Fisch, S.M. Making Educational Computer Games "Educational". Proceedings of IDC '05, 56-61.

18. Ferster, C.B., Skinner, B.F., Cheney, C.D., Morse, W.H., \& Dews, P.B. Schedules of Reinforcement. New York: Appleton-Century-Crofts, 1957.

19. Foxx, R.M., McMorrow, M.J., and Mennemeier, M. Teaching social/vocational skills to retarded adults with a modified table game: an analysis of generalization. Journal of Applied Behavior Analysis, 17 (1984), 343352.

20. Frederick, W.C., and Walberg, H.J. Learning as a function of time. Journal of Educational Research, 73 (1980), 183-194.

21. Garris, R., Ahlers, R. and Driskell, J. E. Games, motivation, and learning: a research and practice model. Simulation \& Gaming, 33 (2002), 441-467.

22. Gee, J.P. What Video Games Have to Teach Us About Learning and Literacy. New York: Palgrave Macmillan, 2003.

23. Gee, J.P. Learning by Design: Good video games as learning machines. E-Learning, 2 (2005), 5-16.

24. Gredler, M.E. Games and simulations and their relationships to learning. In Jonassen, D. H. (2004)

Handbook of Research on Educational Communications and Technology (pp. 571-583). Mahwah, NJ: IEA Publications., 2004.

25. Habgood, M.P.J. The Effective Integration Of Digital Games And Learning Content. PhD Thesis, University of Nottingham, 2007.

26. Herrnstein, RJ. Relative and absolute strength of response as a function of frequency of reinforcement. Journal of the Experimental Analysis of Behavior, 4, 267-272, 1961.

27. Karweit, N. Time-on-task reconsidered: Synthesis of research on time and learning. Educational Leadership, 41 (1984), 32-35.

28. Keller, F. S. Good-bye teacher... Journal of Applied Behavior Analysis, 1, (1968), 79-89.

29. Kiili, K. Educational Game Design: Experiential gaming model revised. Tampere University of Technology, Research report 3, 2005. 
30. Koster, R. A theory of fun for game design. Scottsdale, AZ: Paraglyph Press, 2005.

31. Lee, J., Luchini, K., Michael, B., Norris, C. and Soloway, E. More than just fun and games: Assessing the value of educational video games in the classroom. In Proceedings of CHI 2004, ACM Press (2004), 1375-1378.

32. Lieberman, D. Management of chronic pediatric diseases with interactive health games: Theory and research findings. Journal of Ambulatory Care Management 24, (2001), 26-38.

33. Lindsley, O. R. From Skinner to precision teaching: The child knows best. In J. B. Jordan \& L. S. Robbins (Eds.), Let's try doing something else kind of thing, 111. Arlington, VA: Council for Exceptional Children, 1971.

34. Lindsley, O.R. Precision teaching: discoveries and effects. Journal of Applied Behavior Analysis, 25, (1992a), 51-57.

35. Lindsley, O.R. Why aren't effective teaching tools widely adopted? Journal of Applied Behavior Analysis, 259, (1992b), 21-26.

36. Loftus, G. R., \& Loftus, E. F. Mind at Play: The Psychology of Video Games. New York, NY: Basic Books, 1983.

37. Lovaas, O. I. Behavioral treatment and normal educational and intellectual functioning in young autistic children. Journal of Consulting and Clinical Psychology, 55 (1987), 3-9.

38. Malone, T. W. Toward a theory of intrinsically motivating instruction. Cognitive Science, 5 (1981), 333-369.

39. Malone, T. W., \& Lepper, M. R. (1987). Making learning fun: A taxonomy of intrinsic motivations for learning. In R. E. Snow \& M. J. Farr (Eds.), Aptitute, Learning and Instruction: III. Conative and affective process analyses (pp. 223-253). Hilsdale, NJ: Erlbaum.

40. Malouf, D.B. The effect of instructional computer games on continuing student motivation. Journal of Special Education, 21,(1987), 27-38.

41. Mayo, M.J. Games for science and engineering education. Communications of the ACM, 50 (2007), 31-35.

42. Medland M.B., and Stachnik, T.J. Good-behavior game: a replication and systematic analysis. Journal of Applied Behavior Analysis, 53 (1972), 45-51, 1979.

43. McClean, P., Saini-Eidukat, B., Schwert, D., Slator, B., and White, A. Virtual worlds in large-enrollment science classes significantly improve authentic learning. In, J. Chambers, (ed.) Selected Papers from the 12th International Conference on College Teaching and Learning, (2001), 111-118.
44. Moreno-Ger, P., Burgos, D., Martínez-Ortiz, I., Sierra, J.L., and Fernández-Manjón, B. Educational game design for online education. Computers in Human Behavior, 24 (2008) 2530-2540.

45. Olympia, D. E., Sheridan, S. M., Jenson, W. R., \& Andrews, D. Using student-managed interventions to increase homework completion and accuracy. Journal of Applied Behavior Analysis, 27 (1994), 85-99.

46. O’Neil, H.F., Wainess, R. and Baker, E.L. Classification of learning outcomes: evidence from the computer games literature. The Curriculum Journal, 16 (2005), $455-474$.

47. Pivec, M., and Kearney, P. Games for Learning and Learning from Games. Informatica, 31 (2007), 419423.

48. Prensky, M. Digital Game-Based Learning. McGrawHill, New York, 2001.

49. Rosas, R., Nussbaum, M., Cumsille, P., Marianov, V., Correa, M., Flores, P., Grau, V. Lagos, F., Lopez, X., Lopez, V., Rodriguez, P., Salinas, M. Beyond Nintendo: design and assessment of educational video games for first and second grade students. Computers \& Education, 40 (2003) 71-94.

50. Ruben, B.D. Simulations, Games, and ExperienceBased Learning: The Quest for a New Paradigm for Teaching and Learning. Simulation \& Gaming, 30 (1999), 498-505.

51. Salen, K. and Zimmerman, E. Rules of Play: Game Design Fundamentals. MIT Press, Cambridge, MA, 2004.

52. Saville, B.K., Zinn, T.E., Neef, N.A., Van Norman, N., and Ferreri, S.J. A comparison of interteaching and lecture in the college classroom. Journal Of Applied Behavior Analysis, 39 (2006), 49-61.

53. Skinner, B. F. Science and Human Behavior. New York: The Free Press, 1953.

54. Skinner, B. F. About Behaviorism. New York: Random House, 1974.

55. Skinner E. A. and Belmont, M. J. Motivation in the classroom: Reciprocal effects of teacher behaviour and student engagement across the school year', Journal of Educational Psychology, 85, (1993), 571581.

56. Swartout, W., and Van Lent, M. Making a game of system design. Communications of the ACM, 46 (2003), 32-39.

57. Turnbull, M., Lapkin, S., Hart, D., and Swain, M. Time on task and immersion graduates' French proficiency. In S. Lapkin (ed.), French second language education in Canada: Empirical studies (pp. 31-55). Toronto: University of Toronto Press, 1998. 
